\title{
Las acciones mentales y el problema de las etapas de su formación: siguiendo a Galperin y Talízina
}

\author{
Mental actions and the problems of their formation by stages: \\ following Galperin and Talízina
}

Yulia Solovieva ${ }^{1}$

Luis Quintanar ${ }^{2}$

\begin{abstract}
RESUMO
En el artículo se revisa la concepción de la formación de la acción por etapas como una de las aportaciones más significativas de P.Ya. Galperin y N.F. Talízina para la psicología. De acuerdo con A.I. Podolsky, dicha concepción puede ser comprendida de dos formas: 1) de forma amplia, como una concepción psicológica general y 2) de forma estrecha, como la concepción aplicada a la teoría de la actividad dirigida de enseñanza-aprendizaje escolar. Los autores proponen la unión de ambas posibilidades de la comprensión de esta concepción como una propuesta metodológica general en el estudio del desarrollo. El artículo analiza la posibilidad de considerar a esta concepción como una metodología aún más amplia, que puede abarcar las formas del desarrollo psicológico óptimo y no óptimo, es decir, cuando surge la necesidad de promover las vías alternas del desarrollo en casos de alteraciones en el sistema nervioso central. Se plantea la necesidad de revisar las etapas de la formación de la acción, así como de los elementos estructurales de las acciones. Se establece la necesidad de considerar los tipos de acciones que se puedan realizar en diversas etapas formativas, tales como la acción práctica y la acción intelectual. Se discute la utilidad de esta concepción psicológica para la metodología de la evaluación y de la corrección neuropsicológica que conduce al desarrollo del niño.
\end{abstract}

Palavras-chave: Teoría de Galperin. Formación de la acción. Psicología educativa. Orientación. Planos de la acción.

\begin{abstract}
The article revises conception of formation of the action by stages as one of the most significant contributions of P.Ya. Galperin and N.F. Talízina for psychology. According to A.I. Podolsky, this conception might be understood in two ways: 1) in a broad way as general psychological conception and 2) in a straight way as conception of the process of teaching and learning according to activity theory. The authors of the article propose to consider the union of these two conceptual possibilities as a general methodological proposal for the study of development. The article analyses the possibility for usage of these methodology in a broad way, which might include modes of positive development together with developmental difficulties. This methodology might be useful also in cases of necessity for creation and implementation of alternative ways for development as in cases of negative functional state of central nervous system. The authors propose the necessity of revision of the stages of formation of the action and of its structural components. The article opens the question about types of actions, which might be considered during formation process, such as practical and intellectual actions. The authors discuss the usefulness of this psychological conception for the methods of assessment, correction and teaching, which conduct of development of the child.
\end{abstract}

Keywords: Galperin's theory. Formation of action. Educational. Orientation. Plans of action.

\footnotetext{
1 Universidad Autonoma de Tlaxcala. ORCID: https://orcid.org/0000-0001-5610-1474.E-mail: yulia.solovieva@correo.buap.mx.

2 Universidad Autonoma de Tlaxcala. ORCID: https://orcid.org/0000-0002-9758-1467. E-mail: ranatniuq@mex.com.
} 


\section{Introducción}

L.S. Vigotsky estableció el proceso de interiorización como una vía posible para el desarrollo cultural del niño, escribiendo que cada función psicológica aparece en la escena dos veces: primero como una función externa, compartida social y, más adelante, como una función interna, individual, ideal (VIGOTSKY, 1996). A este proceso de cambios de las acciones y de los conceptos, desde el plano material y compartido hacía el plano ideal e independiente, se le llama proceso de interiorización.

Este proceso debe ser comprendido como la vía del desarrollo psicológico cultural del niño en la sociedad humana. Al mismo tiempo, surge la posibilidad de analizar las dificultades y los problemas en esta vía desde el punto de vista de diversos obstáculos que surgen en el proceso de interiorización. Es posible decir que, si el psicólogo logra correctamente identificar los obstáculos en la interiorización, podrá, a su vez, buscar las vías efectivas para la superación de dichos obstáculos. De esta forma el término de interiorización sirve, al mismo tiempo, como una caracterización teórica del desarrollo histórico-cultural y como una metodología práctica para estudios psicológicos concretos, relacionados con el desarrollo infantil en condiciones óptimas y ante sus diversas desviaciones.

Debemos subrayar que, definitivamente, se trata de una vía posible y relativa, y no de una vía obligatoria y absoluta. El desarrollo cultural del niño siempre debe ser comprendido, precisamente, como una posibilidad, debido a que las condiciones necesarias para esta interiorización, por diversas razones, pueden no darse en la vida concreta de cada niño. La frase citada de L.S Vigotsky hace someter a duda toda la posición clásica en la psicología acerca de un mundo psíquico interno, encerrado en la conciencia o en el cerebro del niño desde su nacimiento. La psique y la conciencia inicialmente existen en la sociedad histórica y, solamente más tarde, puede surgir, por segunda vez, en la psique individual del niño.

$\mathrm{Al}$ introducir esta comprensión no clásica del desarrollo del niño, Vigotsky no alcanzó a profundizar y mostrar con detalles la vía de esta interiorización. Los seguidores de este autor han trabajado sobre diversos aspectos de esta vía. 
Uno de estos aspectos, en especial, es la formación de la acción desde el plano material hacia la acción en el plano ideal. Esta vía se incluye en la concepción de la formación de la acción mental de P.Ya. Galperin y N.F. Talizina. Queremos señalar que, a pesar de que P.Ya. Galperin indudablemente se considera creador de esta concepción y con su nombre se relaciona una comprensión amplia de la misma como una concepción psicológica teórica general. Galperin (2000) escribe que, para la formación de la acción ideal, previamente se debe conformar la acción material correspondiente. El encuentro de una acción material adecuada junto con las condiciones que garanticen su óptima formación, representa un objetivo esencial del estudio psicológico. A este sistema de condiciones en las cuales la acción debe ser formada, Galperin la denomina teoría de la formación de la acción por etapas. "Nosotros elegimos el sistema de condiciones que garantiza la formación de esta acción de una forma causal, con las características preestablecidas. Este sistema de condiciones conforma lo que llaman la teoría de la formación por etapas" (GALPERIN, 2000, p. 32-33).

N. F. Talizina, por su parte, aportó la segunda aplicación de esta concepción a la teoría de la actividad de enseñanza-aprendizaje. Lo anterior significa que Talizina estudió con detalle las condiciones ante las cuales se deben formar las acciones intelectuales durante el proceso de enseñanzaaprendizaje escolar para el ámbito de la escuela primaria, secundaria y preparatoria. Resulta importante e interesante reflexionar sobre las aportaciones de estos dos célebres autores de nuestra época, encontrando las semejanzas y las diferencias entre estas propuestas, así como sus aportaciones para la ciencia psicológica (SOLOVIEVA; QUINTANAR, 2018).

El objetivo del presente artículo es comentar las aportaciones de P.Ya. Galperin y N.F. Talizina de una forma particular y proponer la necesidad de precisar y determinar, tanto las etapas de la formación de la acción por etapas, como la forma de las acciones y los elementos que participan en este proceso. 
2 P. Ya. Galperin. La concepción de la formación de la acción mental por etapas como una concepción psicológica general

Uno de los elementos clave de la teoría de Galperin (2000) es la propuesta de estudiar procesos psicológicos en lugar de fenómenos psicológicos. Defendiendo esta postura, expresó que ninguna ciencia se puede consolidar si su objeto de estudio se limita a fenómenos o características. Tales características son los fenómenos que normalmente aparecen en todos los textos de psicología como categorías básicas: memoria, atención, pensamiento, lenguaje, emociones. Estas categorías aparecen como características o elementos de la conciencia o como propiedades del cerebro (lo cual, en ocasiones, se comprenden como sinónimos), pero pocos psicólogos han logrado imaginar o establecer la génesis, la naturaleza, o la vía del desarrollo de estos fenómenos. Podemos decir también que estos fenómenos se postulan, pero no se estudian como tal.

Galperin (2000), propone que, precisamente, los procesos psicológicos deben conformar el objeto de la ciencia psicológica. Esta idea es muy cercana y obvia desde la concepción de Vigotsky acerca de la interiorización que nos hace comprender que los procesos no están acabados, que no pueden consolidarse desde el inicio, sino que pasan por complejas transformaciones. Los psicólogos del desarrollo deben estudiar estas transformaciones. Todos los procesos surgen a partir de la colaboración, de la participación colectiva, surgen en la zona del desarrollo próximo, la cual se transforma en la zona del desarrollo actual "el mañana" del desarrollo psicológico del niño.

Galperin (2000) fue más adelante en la concepción del desarrollo y expresó que no solo es posible estudiar los procesos durante su formación, sino que, además, conociendo las condiciones óptimas para esta formación, es posible influir positivamente sobre ellos. De esta forma se proclamaba una nueva psicología, "con la comprensión del contenido concreto de la psique humana, con la relación entre la psique y el cerebro, el origen y el desarrollo de la psique humana y con los tipos y formas de la actividad psíquica y las regularidades de su conformación durante la vida” (PODOLSKY, 2000, p. 10). 
¿Cómo podemos comprender esta nueva psicología? En una ciencia nueva se establecen nuevos objetivos, nuevos principios, nuevos términos. Justamente es lo que proponía Galperin con su concepción de la formación de la acción mental por etapas.

Cómo llamar aquello que se desarrolla? Los términos y las categorías comunes ya no sirven para este propósito, porque toda la psicología cambia como ciencia: cambia su objeto de estudio, cambia su unidad de análisis y cambia su método.

No toda la acción debe constituir el objeto del estudio psicológico, sino solo la orientación en toda acción humana. Debemos comprender con ello que la acción humana siempre es un proceso y no un fenómeno.

Para diferenciar a la acción de otros procesos y fenómenos, es necesario recordar las características esenciales de la acción cultural humana. La acción se presenta como un problema en ciertas condiciones (PETUKHOV, 2009), la acción siempre está motivada (LEONTIEV, 1984), siempre es subjetiva (RUBINSTEIN, 2000) y siempre se dirige a un objetivo (LEONTIEV, 1984). En otras palabras, también se puede decir que una persona siempre desea solucionar un problema o realizar una acción dada. Ejemplo psicológico de acciones puede ser que un niño desea dibujar algo (SOLOVIEVA; QUINTanar, 2016 a) o que unos niños en la escuela primaria se interesan por la solución de un problema matemático (ROSAS; SOLOVIEVA; QUINTANAR, 2017).

La acción humana se presenta siempre en ciertas condiciones y con ciertas características y estas condiciones y características pueden analizarse objetivamente. Este análisis objetivo de las características permite que las características deseadas de la acción se introduzcan desde el inicio mismo de su formación. Desde el punto de vista de Galperin (2000), las acciones pueden formarse en el sujeto psicológico, en diferentes edades y en diversas áreas del conocimiento. El éxito depende de un estudio psicológico previo experimental que permite analizar con detalle las características deseables y probables de la acción que se pretende formar.

En cuanto a los planos de la realización de la acción y sus etapas viables, Galperin (2000) estableció cuatro etapas fundamentales: (1) acción material o materializada (cuando la acción se realiza con el uso de las manos y apoyos 
externos); (2) perceptiva (cuando la acción se utiliza solo con el apoyo visual o apoyo de los ojos); y (3) acción verbal externa o externa para sí (pronunciación sin su vocalización) y (4) la acción interna (mental). Así, una misma acción debe pasar por todas las etapas señaladas, es decir, siempre se debe tratar de una misma acción. Un ejemplo de esta acción puede ser la acción de solución de un problema en matemáticas. A su vez, el ejemplo de conducir una bicicleta no puede ser ejemplo, debido a que no se puede conducir una bicicleta en un plano mental. Esta acción se puede automatizar, pero no puede interiorizarse o pasar al plano mental.

Para la formación dirigida de la acción, Galperin (2000) propone cinco etapas, en las cuales, a las cuatro formas de la existencia probable de una acción, se le agrega la primera etapa: etapa motivacional. Estas cinco etapas formativas son:

1) Etapa motivacional, que pretende garantizar y despertar un interés o una necesidad para el sujeto.

2) Etapa material o materializada, cuando la acción se realiza con ayuda de objetos concretos o esquemas simbólicos externos.

3) Etapa perceptiva, que se debe realizar con los ojos, pero sin apoyos materializados.

4) Etapa verbal, cuando el sujeto explica en voz alta todo lo que hace; etapa verbal en silencio, cuando el sujeto explica en su lenguaje interno todo lo que hace.

5) Etapa mental, cuando la acción es eficaz, rápida, sin apoyos y sin pronunciación.

Debido a lo anterior, la parte compleja de esta concepción de la formación de la acción mental por etapas es que en ella solo se consideran las acciones intelectuales que se presentan como problemas que en un principio pueden pasar al plano mental.

Esta misma complicación lleva a pensar que en realidad, se debe especificar que no se interioriza toda la acción, sino solo su parte de orientación. Esta posición no siempre fue expresada con claridad por parte de Galperin y sus seguidores. Podemos imaginar que se daba por el hecho de que esto ya estaba conocido, pero, sin duda, la ausencia de esta aclaración puede llevar a diversos errores y contradicciones. Por ejemplo, una de las contradicciones es el nombre mismo de la concepción que debería decir: formación de la orientación por etapas 
en lugar de formación de la acción por etapas. Esta modificación conceptual permitiría generalizar la concepción de Galperin e incluir, sin ninguna dificultad, a todas las acciones, incluyendo a las acciones prácticas, intelectuales, deportivas, artísticas, cotidianas, etc. Es indudable que, para estudios psicológicos más amplios, incluyendo los estudios que se realizan en la psicología del desarrollo, la neuropsicología infantil y la neuropsicología del adulto, la inclusión de diversos tipos de acciones con la concepción de interiorización de la orientación es sumamente útil (SOLOVIEVA; QUINTANAR, 2019).

\section{N.F. Talizina. La concepción de la formación de la acción mental por etapas como una concepción en la teoría de la actividad de la enseñanza-aprendizaje}

N.F. Talizina desarrolló la aplicación particular de la concepción general de Galperin específicamente para la actividad de enseñanza-aprendizaje escolar. La misma autora siempre expresó que el objetivo de su vida fue mostrar una posible modificación de todo el proceso de enseñanza-aprendizaje en base a la teoría de Galperin.

Una de las aportaciones teóricas de esta autora es el hecho de señalar la estrecha relación entre el concepto de zona de desarrollo próximo (ZDP) de L.S. Vigotsky y el concepto de base orientadora de la acción (BOA) de P.Ya. Galperin. Esta idea no la expresó claramente el mismo Galperin, sino Talizina. En diversos trabajos experimentales, realizados con sus alumnos en Rusia (KARPOV; TALIzina, 1986, 1989), Vietnam, China y México (TALIZINA, 2018; SOLOVIEVA, 2004, 2014), se ha mostrado que la ZDP puede ser valorada a partir del nivel en el cual se introduce la BOA intelectual: materializado, perceptivo o verbal oral.

Talizina mostró también que no necesariamente cada acción debe pasar por todas las etapas formativas, es decir, no necesariamente se debe iniciar con la acción material o materializada, sino que se puede iniciar, por lo menos, desde la acción perceptiva. Todo depende del nivel previo en el que el niño se encuentra, lo 
cual puede ser diagnosticado a través de metódicas especiales (KARPOV; TALIZINA, 1986, 1989; SOLOVIEVA, 2014).

Los datos de estos trabajos experimentales sugieren que la ZDP se puede valorar con precisión y no como algo meramente descriptivo. La BOA es más amplia si el niño realiza la acción con ayuda verbal y más estrecha si el niño requiere de ayudas perceptivas o materializadas (TALIZINA; KARPOV, 1987).

Estos trabajos experimentales también señalan, siguiendo la idea de Galperin (2000), que la BOA puede ser de diferentes tipos, en dependencia de sus características. Se muestra que, para una óptima ejecución de una tarea intelectual, es necesario considerar todas las características necesarias y suficientes de la acción que se va a realizar y establecerlas como una objetividad dada para el sujeto en el esquema de la BOA. En diversos trabajos de Talizina y sus colaboradores se mostraron diseños de esquemas de la base orientadora para diferentes temas y materias en la escuela primaria, secundaria y preparatoria (TALIZINA, 1984, 2019, 2018; TALIZINA; SOLOVIEVA; QUINTANAR, 2017).

En relación con las etapas de la formación de la acción mental, se puede notar una diferencia importante respecto al planteamiento de Galperin (2000). Talizina (2019) diferencia más claramente la acción material y materializada y diferencia a ambas acciones de la acción perceptiva. Los autores de este artículo opinamos que esta precisión fue posible a partir de la realización de múltiples estudios de Talizina (y de sus alumnos y colaboradores) con niños escolares menores, al inicio del proceso de aprendizaje escolar. En primer lugar, es necesario mencionar las aportaciones de N.G. Salmina, quien mostró profundas diferencias entre la ejecución de la acción con objetos reales y sus sustitutos en la edad fronteriza preescolar y escolar, así como las dificultades que los niños pueden presentar con el uso de los objetos simbólicos (Salmina, 1988).

Es posible, por lo tanto, pensar en una ampliación de las etapas de realización de una acción intelectual se puede expresar de la siguiente manera:

- $\quad$ Acción material, que se realiza con los objetos reales (concretos).

- Acción materializada, que se realiza con los sustitutos materiales de los objetos reales (fichas o signos externos). 
- $\quad$ Acción perceptiva, que se realiza con ayuda de dibujos y/o esquemas, pero sin el uso de objetos o símbolos externos.

- $\quad$ Acción verbal externa, que puede ser oral o escrita.

- $\quad$ Acción verbal en silencio.

- Acción mental.

Talizina (2009) realiza otra modificación al ubicar la etapa motivacional fuera del proceso de la formación de la acción por etapas y colocarla como una de las cuatro etapas del proceso de organización del aprendizaje, cuyas etapas son:

- Etapa motivacional.

- Etapa de elaboración de la BOA.

- Etapa de materialización de la acción, que puede incluir la realización de la acción en su forma material, materializada, perceptiva con su interiorización hasta la etapa de acción verbal y mental.

- Etapa de verificación y control.

En nuestra opinión, una de las principales dificultades de esta concepción es considerar a las acciones únicamente intelectuales, relacionadas con las materias escolares y con las acciones lógicas. Dicha situación puede provocar una apreciación un tanto reducida de las posibilidades de esta importante concepción teórica, restringiéndola únicamente al ambiente escolar y a edades psicológicas particulares (a partir de la edad del aprendizaje escolar dirigido).

$\mathrm{Al}$ mismo tiempo, queremos subrayar que las posibilidades de esta concepción psicológica son mucho más amplias, a lo que dedicaremos nuestro siguiente apartado.

\section{Revisión de la concepción de la formación de la acción}

\section{A. Elementos de la acción}

De acuerdo con Galperin (2000), en la acción es posible identificar tres partes funcionales: la orientación, la ejecución y el control y la verificación.

La unidad que se interioriza, en la concepción de Galperin, es la acción. Si no se precisa de qué acción se trata, no se puede hablar de una interiorización. Al mismo tiempo, para Galperin, la acción siempre es un problema, por lo que 
siempre se trata de acciones intelectuales. Este punto es fundamental para comprender la teoría de Galperin, porque no todas las acciones se pueden interiorizar en su totalidad. Muy frecuentemente, se quedan diversos elementos que permanecen externos, corporales o perceptivos en las acciones prácticas, deportivas, artísticas. En el caso de todas estas acciones podemos hablar de automatización, como de una de las variantes de su apropiación cultural, pero no de su formación por etapas en todo el sentido de la palabra.

Por lo tanto, desde un inicio es necesario precisar que se trata de la interiorización de la parte de orientación de todos los tipos de acciones que se pueden considerar. Evidentemente existen diversos tipos de acciones que se pueden diferenciar por su objeto y por su producto. Por lo menos, resulta útil mencionar que existen acciones prácticas, intelectuales, comunicativas, deportivas y artísticas. No hablaremos en este escrito sobre otros tipos probables de acciones. Lo importante es constatar que ningún tipo de acción se puede realizar sin su orientación.

La parte de orientación, en psicología, representa el mayor interés, debido a que precisamente de ella depende la ejecución exitosa de la acción en casos comunes. Un elemento clave de esta orientación es el concepto de la base orientadora de la acción introducido en la psicología por Galperin (1998) y desarrollado por Talizina (1984, 2019).

La base orientadora de la acción (BOA) es aquella información en la que se basa el sujeto, de forma consciente o inconsciente, cuando realiza una acción. Una de las opciones es cuando el sujeto es guiado de forma óptima para la realización de esta acción, en cuyo caso se le presenta un esquema de la BOA, de lo cual el sujeto tiene que ser ya consciente, por lo menos parcialmente.

Este último punto es de gran interés para la organización de estudios psicológicos. Galperin (2000) enfatizó que la ejecución debe ser consciente para el propio sujeto y que esta característica implica que él pueda explicar con sus propias palabras sobre esta acción. En este punto surge una duda metodológica sobre la posibilidad de aplicar este concepto a niños muy pequeños o a niños con severas dificultades en su desarrollo, ya que no serían capaces de expresar con palabras lo que ellos realizan, lo cual no descarta la posibilidad de realizar correctamente las 
acciones con la ayuda del adulto. Este es un punto de discusión que, a nuestro parecer, puede y debe ser atendido con ayuda de estudios de la psicología del desarrollo y de la neuropsicología (SOLOVIEVA; PELAYO; QUINTANAR, 2016).

Consideramos que, indudablemente Galperin (2000) tiene razón en que el trabajo con el esquema orientador debe ser consciente para el sujeto, pero no tiene razón en que el indicador de esta consciencia sea solamente que el sujeto hable de lo que hace. Tal proposición contradice a la teoría misma de la formación de las acciones por etapas: una adecuada ejecución de la acción en nuevas condiciones puede ser un adecuado indicador de que la realización de dicha acción es consciente para el sujeto y que el sujeto comprende lo que hace. Lo que definitivamente debe evitarse es la repetición de una misma acción en las mismas condiciones, pero justamente es lo que hacen todos los maestros. Tal mecanización no conduce a la conciencia, a la generalización y a una ejecución racional y no permite que la acción pase a su forma más superior, siempre y cuando se trate de acciones intelectuales.

La presentación del esquema de la BOA implica la elaboración de una imagen de toda la situación en la cual se realiza la acción, de las condiciones óptimas para su realización, el contenido, las operaciones que se requieren y el plan de ejecución con un control constante (GALPERIN, 2000). La BOA es lo que permite la correcta realización de la acción que se plantea.

La BOA incluye, necesariamente, la representación de una imagen general de la tarea o de la situación que implica la resolución, el análisis de los elementos de dicha situación, la realización gradual de la ejecución de la acción con la posibilidad de verificar y corregir todo el proceso desde su inicio. Todo el proceso debe ser comprensible y reflexivo para el sujeto que lo realiza, por lo que excluye una ejecución mecánica. Precisamente estas características deben tomarse en cuenta para promover el concepto de la BOA para las situaciones de trabajo con la comprensión y la producción del lenguaje en la edad escolar.

De acuerdo con Galperin (1998), si consideramos sus características principales, pueden existir diferentes tipos de BOA: el contenido, el carácter de la presentación y las posibilidades de aplicación. Respecto a su contenido, la BOA 
puede ser completa o incompleta, es decir, puede contener toda la información necesaria para resolver la acción o bien, esta información puede faltar. En relación con el carácter de la presentación, la BOA puede ser independiente (elaborada por el mismo sujeto) o dependiente (presentada por otro sujeto). A pesar de que en la escuela es bastante complejo obtener la forma independiente de la BOA, es posible intentarlo. El maestro puede inducir a los alumnos a la formulación de las reglas, incluso a la elaboración de la orientación y de las reglas.

El esquema de la BOA se debe elaborar considerando las tres características fundamentales. Al igual que la misma acción, el esquema de la BOA se puede presentar en diversos planos: material, materializado, perceptivo, verbal y, finalmente, se puede interiorizar. Debido a lo anterior, parece útil hablar de la interiorización de la parte de la orientación de la acción, en lugar de toda la acción en general, especialmente para las acciones prácticas, comunicativas, artísticas y deportivas, en las cuales no es factible un plano mental de su realización.

\section{B. Planos de interiorización de la acción}

Como se ha expresado anteriormente, la teoría de la interiorización gradual de las acciones intelectuales permite garantizar el paso de las acciones del mismo tipo con contenido diverso al nivel cada vez más interno. En la concepción de la formación de las acciones por etapas se pueden establecer los niveles consecutivos, desde el nivel más externo, hasta el plano más interno para diversas acciones: material, materializada, perceptiva, verbal externa, verbal escrita, interna. Para Galperin (2000), la etapa interna implica dos posibilidades: operaciones con las imágenes ya conformadas de los objetos y/o con conceptos internos. Galperin escribe que cada imagen es producto de una acción y "no tanto de la acción ideal, sino de la acción real con aquellos objetos que después se representan ante nosotros como imágenes" (GALPERIN, 2000, p. 26).

En este texto queremos expresar la necesidad de reconsiderar las etapas de interiorización de la acción (o de la orientación dentro de una acción) que se proponen para el plano de las imágenes. Resulta que nuestra propia práctica experimental y clínica ha permitido diferenciar dos planos o formas (que al 
mismo tiempo son dos etapas) perceptivas. Esta división no se señala en los textos de Galperin y Talizina, quienes hablan de una única acción perceptiva o de la acción con imágenes, a la cual proponemos dividir en dos tipos de acción perceptiva. La división depende del objeto que se percibe por parte del sujeto: imagen concreta o imagen generalizada (esquemática o simbólica).

En primer lugar, se puede hablar de la presencia de percepción y la realización de una acción de reconocimiento o de clasificación de imágenes concretas, tales como tarjetas, fotografías, dibujos de muebles o de animales. En segundo lugar, se puede hablar de otra situación, cuando se trata de una acción de reconocimiento o clasificación de imágenes abstractas como tablas, gráficas, mapas, fichas, esquemas, etc. Es indudable que para un niño la primera acción perceptiva de imágenes concretas es mucho más accesible que la segunda acción perceptiva de esquemas y gráficas.

El ejemplo 1 muestra la diferencia entre una imagen concreta y una imagen abstracta o esquematizada. En este ejemplo se puede observar cómo el maestro del nivel preescolar dibujó diferentes personajes de juego temático de roles y utilizó la simbolización esquemática (con figuras geométricas) para señalar qué tarjeta le tocaría a cada participante del juego para elegir su rol. En este ejemplo, a los pasajeros les tocaría una tarjeta cuadrada, etc. 
Ejemplo 1 - Dibujos y símbolos en el pizarrón.

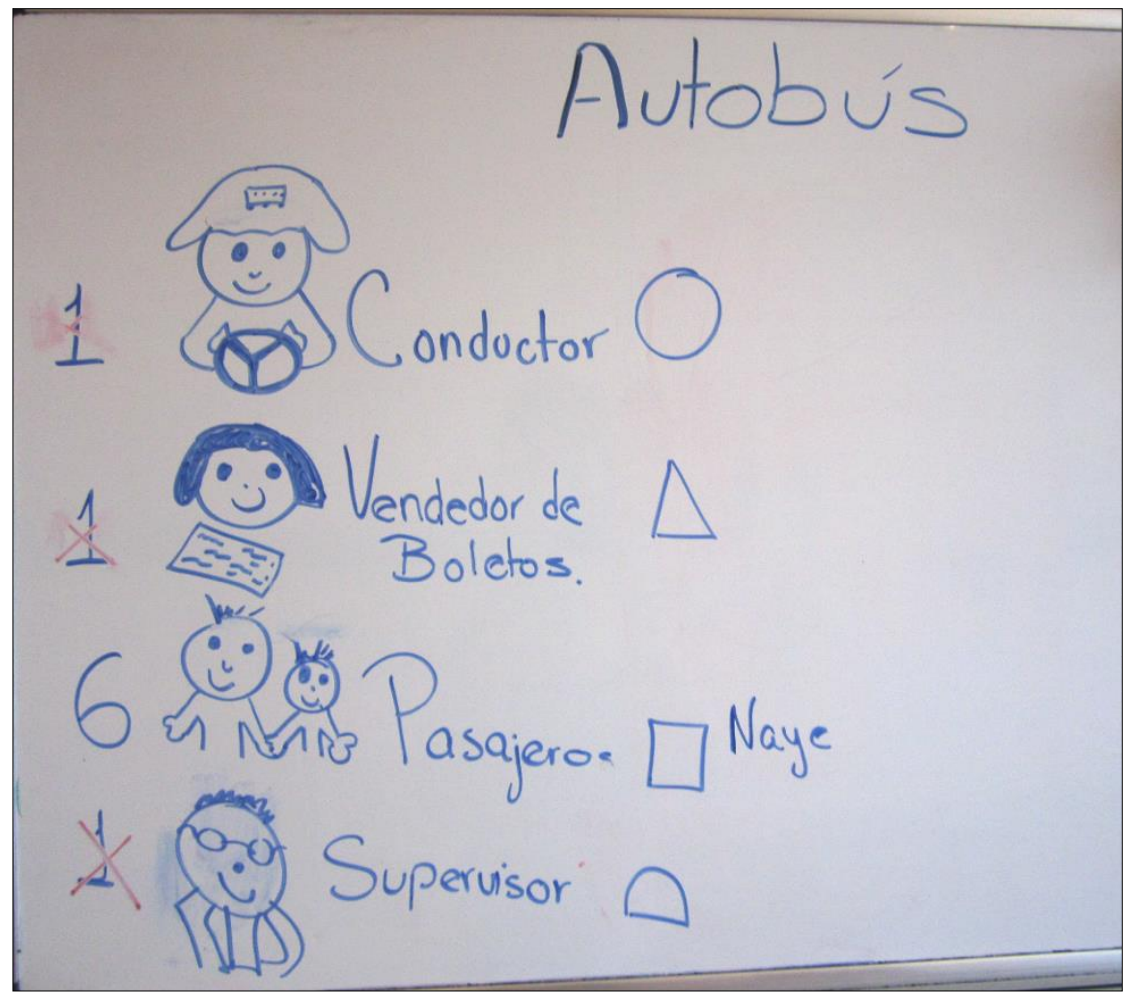

Nuestra experiencia de aplicación de procedimientos de evaluación y formación, en México, muestra una diferencia psicológica fundamental, cuando un niño trabaja con la imagen concreta o con la imagen esquemática. Especialmente se trata de menores que presentan diversos problemas en su desarrollo (SOLOVIEVA; QUINTANAR, 2016b).

Una diferencia similar se ha mostrado, en Rusia, en los trabajos de Salmina y sus colaboradores en el caso de uso de objetos y juguetes y sus substitutos por parte de niños preescolares (SALMINA, 1988). De acuerdo con nuestros estudios, en México y en Colombia, el adulto debe preparar y conducir al niño para el uso de los substitutos y hacerlo dentro de la actividad rectora de la edad preescolar: el juego temático de roles (BONILLA; SOLOVIEVA, 2016; GONZÁLES; SOLOVIEVA, 2016). Así como al niño le cuesta trabajo pasar del objeto a su substituto, también le cuesta trabajo pasar de la imagen concreta a la imagen simbólica, especialmente cuando no hay una base sólida previa para ello, es decir, en los casos de una pobre preparación para los estudios escolares (Solovieva et al., 2015) o alteraciones en el sistema nervioso central 
(SOLOVIEVA; QUINTANAR, 2015, 2016a). Consideramos que se deben diferenciar claramente la acción material de la acción materializada, así como la acción perceptiva concreta de la acción perceptiva esquematizada o simbólica. Algo similar sucede cuando hablamos del plano de la BOA, la cual también puede ser presentada al sujeto en uno de los planos señalados.

De esta forma, proponemos considerar las siguientes etapas (planos) de la formación de la acción o de alguno de sus componentes estructurales, como puede ser el objeto, el resultado y la orientación:

- $\quad$ Acción material que se realiza con los objetos reales (concretos).

- Acción materializada que se realiza con los sustitutos materiales de los objetos reales (fichas o signos externos).

- $\quad$ Acción perceptiva que se realiza con la ayuda de dibujos.

- Acción perceptiva que se realiza con la ayuda de esquemas representados gráficamente.

- $\quad$ Acción verbal externa, que puede ser oral o escrita.

- $\quad$ Acción verbal en silencio.

- $\quad$ Acción mental.

Finalmente, cabe recordar que la acción del sujeto se realiza siempre en el plano de la consciencia, siempre existe reflexión del objetivo de esta acción, sin importar de cuál de los planos se trate. La realización mecanizada o inconsciente, la imitación o el reflejo, no puede considerarse como una acción.

\section{Secuencia de los planos de la interiorización}

Además de señalar los planos existentes de la acción, es importante considerar la secuencia de los planos de la acción durante el proceso formativo, incluso en el proceso de la evaluación del desarrollo y del éxito del proceso de enseñanzaaprendizaje en la escuela. Como lo hemos expresado, Galperin (2000) señala que la acción primaria es la acción material con los objetos reales. La siguiente posibilidad para Galperin, es la acción materializada que se realiza con los substitutos de los objetos o la acción perceptiva. Sin embargo, en los textos de Galperin (1998, 2000) no 
se precisa con claridad la secuencia de las formas de la acción y Talizina (2019) siempre señaló que la acción materializada antecede a la acción perceptiva.

Debemos expresar que la elección del nivel adecuado para iniciar con el proceso formativo depende de muchos factores, y no se debe pensar que el orden de los planos de la acción mecánicamente se repite en todos los casos. La elección del nivel de la acción con que el experimentador puede iniciar depende de la edad del participante, del grado de dificultad que presenta o del eslabón funcional del sistema nervioso central que puede estar comprometido. También depende del tipo mismo de la acción, si se trata de una acción práctica, comunicativa, deportiva, artística, o de una acción intelectual.

Cabe señalar que en los textos de Galperin y Talizina, se trataba, en la mayoría de los casos, de acciones intelectuales, debido a que la formación de estas representaba el foco de interés profesional de estos autores. Sin embargo, diversos focos de interés pueden ser identificados en el proceso formativo. Uno de ellos es la necesidad de elaborar programas de corrección para niños con problemas en el desarrollo y en el aprendizaje escolar, después de la evaluación neuropsicológica. En estos casos, el éxito de las sesiones de corrección depende, en gran medida, de la preparación conceptual del psicólogo o del educador, quien decide cómo iniciar el proceso formativo.

En estas sesiones, frecuentemente podemos afirmar que el paso por las etapas consecutivas permite garantizar una sólida consolidación de las acciones y permitir que el niño no se estanque en un mismo nivel de realización de las acciones. Además, es posible diferenciar las acciones que se pueden utilizar en las sesiones de corrección en dos grupos amplios: acciones intelectuales y acciones prácticas cotidianas (no intelectuales).

Las acciones intelectuales son aquellas que requieren de un proceso de solución, semejante a la solución de un problema. De hecho, las acciones intelectuales se presentan siempre como un problema a resolver. La presencia de un problema requiere del análisis de las características dadas en determinadas condiciones (PETUKHOV, 2009). Al final de la solución de un problema siempre surge un resultado nuevo, algo que no ha existido antes de la solución. Por ejemplo, 
todas las tareas de clasificación, seriación, identificación de características, son tareas intelectuales. Sus resultados son los grupos obtenidos o seleccionados de objetos, características, conceptos, etc. Sin pretender mostrar todas las posibilidades, ejemplificamos acciones intelectuales que pueden ser útiles y aplicables durante el proceso de corrección y desarrollo en las edades infantiles. Debemos señalar que las últimas etapas de los planos internos no son aplicables durante los procedimientos de corrección y deben comprenderse como metas (lejanas y cercanas) del desarrollo intelectual futuro (próximo) de cada niño y adolescente.

A continuación, presentaremos ejemplos de acciones intelectuales y cotidianas, prácticas y comunicativas en diferentes niveles o planos de la formación de la acción. Nos parece importante señalar que para Galperin (2000) las acciones perceptivas eran acciones ideales, junto con las acciones mentales. Las acciones externas para Galperin son aquellas que se realizan con los órganos propios (cuerpo), como las manos o los órganos articulatorios. Para evitar confusiones, en la tabla 1 agregamos, junto con la mención del plano de cada acción, su forma global que, para Galperin, puede ser externa o interna.

En los ejemplos de la tabla 1 se puede observar que se trata de acciones lúdicas, comunicativas, de reconocimiento o de imaginación reproductiva. Pero en el caso de las acciones intelectuales siempre se trata de la solución de un problema que no se presenta en condiciones externas dadas, sino que requiere de un análisis previo de los datos. Es posible observar también que las acciones cotidianas no requieren del análisis de las condiciones o datos y son acciones más directas con objetos externos, perceptivos e internos. 
Tabla 1 - Ejemplos de acciones cotidianas/comunicativas en diversos planos de la actividad.

\begin{tabular}{|c|c|c|}
\hline Plano de la acción & $\begin{array}{c}\text { Acciones cotidianas, } \\
\text { prácticas y } \\
\text { comunicativas }\end{array}$ & Acciones intelectuales \\
\hline Material / externa & $\begin{array}{l}\text { Peinarse, comer con } \\
\text { cuchara, acostar a una } \\
\text { muñeca. }\end{array}$ & $\begin{array}{l}\text { Agrupación de objetos reales } \\
\text { por una o dos } \\
\text { características. }\end{array}$ \\
\hline Perceptiva concreta / ideal & $\begin{array}{l}\text { Identificar o reconocer } \\
\text { dibujo de un animal o una } \\
\text { persona conocida en } \\
\text { fotografía }\end{array}$ & $\begin{array}{l}\text { Agrupación de tarjetas, } \\
\text { fotografías e imágenes por } \\
\text { una o dos características. }\end{array}$ \\
\hline Materializada / externa & $\begin{array}{l}\text { Sustituir la cuchara por un } \\
\text { palito en el juego. }\end{array}$ & $\begin{array}{l}\text { Agrupación de figuras } \\
\text { geométricas. }\end{array}$ \\
\hline $\begin{array}{c}\text { Perceptiva esquematizada / } \\
\text { Ideal }\end{array}$ & $\begin{array}{l}\text { Reconocer señales de } \\
\text { tránsito. }\end{array}$ & $\begin{array}{l}\text { Realizar análisis fonológico } \\
\text { de sonidos en las palabras } \\
\text { con ayuda de esquemas } \\
\text { dibujados. }\end{array}$ \\
\hline Verbal oral /externa & $\begin{array}{l}\text { Participar en un diálogo } \\
\text { cotidiano sobre el tema del } \\
\text { clima. }\end{array}$ & $\begin{array}{l}\text { Identificar a los personajes y } \\
\text { sus acciones del cuento } \\
\text { leído. }\end{array}$ \\
\hline Verbal escrita /externa & Poner fecha por escrito. & $\begin{array}{l}\text { Elaborar oraciones de } \\
\text { manera independiente. }\end{array}$ \\
\hline Lenguaje en silencio & $\begin{array}{l}\text { Recordar el nombre de una } \\
\text { persona. }\end{array}$ & $\begin{array}{l}\text { Solucionar problemas } \\
\text { matemáticos. }\end{array}$ \\
\hline Mental / interna & $\begin{array}{l}\text { Imaginar y recordar los } \\
\text { sucesos del día. }\end{array}$ & $\begin{array}{l}\text { Solucionar un problema } \\
\text { matemático. }\end{array}$ \\
\hline
\end{tabular}

Lo interesante en la postura de Galperin (1998), es que las acciones intelectuales no se conforman como internas, ideales, sino que se pueden establecer en los planos externos. "El paso desde el plano externo hacia el interno y la transformación de las acciones objetales reflejadas en los fenómenos psicológicos, se representa como la vía de la formación de las acciones mentales, en el sentido amplio, acciones ideales. El papel de la BOA, el éxito de su realización y una aplicación nueva, se explica en parte por la calidad de su "uso constante" y por la diferenciación de los tipos de orientación en el objeto de la acción” (Galperin, 1998, p. 427). 
En la tabla 1 podemos notar que los últimos niveles, internos ideales (plano del lenguaje en silencio y plano mental), no son aplicables al proceso de corrección en casos de dificultades. Estos planos constituyen los resultados del desarrollo de cada niño como producto de su actividad intelectual previa.

Desde el punto de vista de la eficacia de la organización de los procedimientos de la corrección y desarrollo en niños con dificultades, es importante considerar el nivel apropiado inicial de cada niño. Igualmente es importante decidir el uso de las acciones intelectuales o cotidianas en diversos niveles. Con el logro de cada niño es posible gradualmente pasar de una etapa a otra o combinar las etapas accesibles para cada niño en particular.

En los trabajos experimentales de Vigotsky y sus seguidores se señala claramente que del adulto depende qué tipo de conceptos va a adquirir el niño y con qué grado de éxito, estabilidad, generalización y funcionalidad. En base a la teoría de la formación de las acciones por etapas, podemos agregar a esta idea que las ayudas pueden realizarse en diversos planos de la acción. La eficacia depende del plano que el elija para cada niño o grupo de niños en un procedimiento formativo o correctivo concreto.

\section{La teoría de la formación de la acción por etapas para el ámbito escolar}

Vigotsky (1996) también expresó la necesidad de considerar cada edad psicológica en el desarrollo ontogenético del niño como un periodo cualitativo que tiene su propio contenido y estructura. Esta idea sobresaliente de Vigotsky hace posible establecer un puente entre la psicología y la pedagogía, porque la psicología puede ayudar a la pedagogía en la escuela, ya que el niño no solamente estudia, sino también se desarrolla. Este desarrollo transcurre en la escuela dentro de una situación particular del desarrollo del niño, en la cual hay dos participantes simultáneos: el colectivo de niños como alumnos de la escuela primaria y el adulto como maestro del nivel correspondiente. El objetivo del proceso educativo en la escuela primaria, de acuerdo a la aproximación de Vigotsky (1991) y sus seguidores (TALIZINA, 2019; DAVIDOV, 2000), es la introducción y la adquisición de 
conceptos científicos de las ciencias básicas: lingüística, matemáticas, ciencias sociales y naturales. Para cumplir con este objetivo, es necesario diferenciar entre conceptos cotidianos (empíricos) y científicos (teóricos).

A diferencia de los conceptos cotidianos, los conceptos teóricos siempre forman sistemas complejos y no se adquieren a través de la simple interacción con los objetos. Si esto fuera así, la humanidad no hubiera necesitado de la ciencia. Frecuentemente se piensa que los conceptos teóricos se construyen espontáneamente sobre la base de la experiencia cotidiana del niño. Este punto de vista se relaciona con la propuesta de Piaget (1953), quien la introdujo a la psicología y a la pedagogía mundial, a pesar de que no haya expresado una visión particular acerca de los objetivos y los métodos de enseñanza. Talizina (2019) escribe que los conceptos teóricos no pueden ser traspados desde el plano verbal desde adulto al niño, sino solo pueden formarse dentro de las acciones intelectuales específicas. Los conceptos teóricos son siempre generalizaciones de objetos y fenómenos a partir de las características necesarias y suficientes según cada area de conocimientos. Estos conceptos conforman sistemas jerrárquicos a diferencia de los conceptos empíricos y cotidianos.

Una alternativa a los métodos tradicionales existentes, es la organización sistémica del proceso de enseñanza-aprendizaje. Entre autores que han aportado al estudio y la aplicación de estos métodos, son los psicólogos representantes de la teoría de la actividad, entre ellos P.Ya. Galperin y N.F. Talizina, reconocidos mundialmente como exponentes de la concepción de la formación de la acción mental por etapas y de la teoría de la actividad aplicada a la enseñanza.

Los conceptos teóricos generales para las matemáticas son el concepto de número y el concepto del sistema decimal (TALIZINA; SOLOVIEVA; QUINTANAR, 2017), mientras que para la gramática son las categorías gramaticales (SOLOVIEVA, 2015, 2016; SOLOVIEVA; QUINTANAR, 2017). A los conceptos teoricos generales, Talizina los denomina como invariantes, subrayando así la idea de que son los conceptos fundamentales de cada area de conocimiento (TALIZINA, 2018). Al mismo tiempo, son los conceptos, con los cuales se debe iniciar proceso formativo. La enseñanza de cada materia se debe 
iniciar con la introducción de estos conceptos invariantes a través de la orientación del maestro y llevar a la formación gradual que garantice el uso de este concepto en las acciones intelectuales externas que corresponden al concepto dado dentro del área dada de conocimiento, hasta lograr su interiorización mediante las acciones correspondientes de los alumnos. Sin entrar en detalles del proceso de interiorización (VIGOTSKY, 1995; GALPERIN, 2000), mencionaremos brevemente la forma de interacción con los alumnos que puede garantizar de mejor forma la organización del trabajo con los conceptos científicos incluidos en las acciones intelectuales de los alumnos.

La acción conserva todas las características esenciales de la actividad y toda su estructura e incluye los elementos estructurales básicos: el motivo, el objetivo, los medios de realización, la base orientadora y el resultado. Las partes funcionales de la acción son: la orientación, la ejecución, la verificación y el control (GALPERIN, 2000; TALIZINA, 2019). Dicha idea es fundamental para la organización de la enseñanza escolar, debido a que no puede existir la enseñanzaaprendizaje sin acciones, es decir, los alumnos siempre deben hacer algo (TALIZINA, 1984). Hacer algo no puede reducirse a escuchar lo que el maestro dice, a copiar del pizarrón, o a memorizar información que no se comprende. Precisamente estas son las conductas que predominan en la escuela el día de hoy.

El maestro en la escuela debe estar preparado para elegir adecuadamente el plano inicial de la acción intelectual que se va a formar, así como comprender la vía para la interiorización de esta acción. Los elementos de la acción escolar, o acción intelectual del alumno, siempre se realizan en uno de los planos de la acción que hemos señalado anteriormente: material, materializada, perceptiva concreta, perceptiva esquemática, verbal.

La elección adecuada del nivel de inicio de la introducción de la acción intelectual, así como la secuencia apropiada de los planos de la acción, significa que el alumno no puede estancarse en un mismo plano de la acción, sino debe ser llevado y acompañado por el maestro para alcanzar el mayor nivel de interiorización, por lo menos de la parte de la orientación de la acción que realiza. Galperin (2000) señaló que no todos los niveles de la acción pueden ser 
interiorizados y que el componente de la acción que más se transforma durante el proceso de interiorización es la BOA. Debido a ello es que, en el proceso de enseñanza se debe pensar en un despliegue máximo de las orientaciones desde los planos externos para garantizar su paso a los planos más internos.

\section{Consideraciones finales}

Dentro de la teoría de la actividad, para Leontiev (1983, 1984, 2003) el elemento central de la acción y de la actividad es su motivo, mientras que para Galperin (2000) es la orientación. Estos puntos de vista de dos grandes psicólogos del signo XX no se deben contraponer, sino unirse dentro de la categoría psicológica de la actividad. Por un lado, no hay actividad sin motivo, pero, por otro lado, no hay resultado de actividad intelectual sin orientación. Tanto el motivo como la orientación, forman parte de la estructura de la actividad. La presencia de la orientación no significa que el proceso no es atractivo para los participantes. Por el contrario, todo proceso de adquisición de conocimientos tiene que ser interesante y atractivo para los alumnos (SOLOVIEVA; MATA, 2017), lo cual no contradice a la idea que debe existir una orientación adecuada para ello. Para que esto sea viable, los maestros deben estar preparados para identificar el sistema de condiciones que permite iniciar la formación de la acción desde los niveles accesibles, como podría ser el nivel material, perceptivo concreto o materializado. No se recomienda iniciar el proceso formativo desde los niveles complejos, como el nivel perceptivo esquematizado o verbal, con niños de escuela primaria. En cada grado escolar, y trabajando con cada grupo de alumnos, el maestro o psicólogo debe tomar la decisión adecuada. Esto solo se puede lograr con el estudio continuo y profundo de la teoría de la actividad y de la concepción de Galperin y Talizina sobre los planos formativos de la acción por etapas. La motivación cognitiva especifica se forma dentro del proceso de enseñanza-aprendizaje dirigido (DAVIDOV, 2000) y conforma una de las etapas de este proceso (GALPERIN, 2000).

Lo anterior concuerda con la postura esencial del paradigma históricocultural acerca del desarrollo intelectual del niño, que señala que, al principio la 
adquisición de los instrumentos psicológicos se da desde el plano externo en las actividades compartidas entre el niño y el adulto. Más adelante, esta interacción se interioriza, lo cual significa que el niño comienza a actuar de forma independiente, individual e interna (VIGOTSKY, 1996).

Todo lo señalado es igualmente válido para la psicología del desarrollo, como para la neuropsicología infantil, siempre y cuando su propósito no se limite a la evaluación de las dificultades (cuantitativa y/o psicométricamente) y a la selección de etiquetas para los niños y sus trastornos. En la neuropsicología histórico-cultural, uno de los métodos principales es el análisis sindrómico, que implica la identificación de la causa de las dificultades, las cuales, desde nuestro punto de vista, incluyen la actividad rectora y el plano de realización de la acción (SOLOVIEVA; QUINTANAR, 2016c). Precisamente uno de los objetivos esenciales de la evaluación neuropsicológica es el diseño y la aplicación de programas de intervención que conducen al desarrollo psicológico del niño y no únicamente "corrigen" sus dificultades. La evaluación neuropsicológica no debe clasificar (o asignarle una patología) al niño, sino insertarlo al proceso de desarrollo formativo (ALVES; GONZÁLEZ; SOLOVIEVA, 2018). Este tipo de evaluación neuropsicológica cualitativa permite disminuir la distancia entre el trabajo del neuropsicólogo y el psicólogo del desarrollo a través de la necesidad de una búsqueda de metodología participativa y formativa. La metodología formativa necesariamente se basa en la concepción de Galperin y Talizina acerca de la formación de las acciones por etapas.

Consideramos que la elaboración y la aplicación de métodos innovadores implican una constante formación y aprendizaje de los docentes y psicólogos que se incluyen en el proceso educativo. La didáctica educativa es una actividad intelectual creativa, en la cual el docente es el principal participante. Podemos afirmar, que la innovación educativa y didáctica modifica no solo a los participantes (niños, alumnos), sino también a los adultos (docentes) de forma cualitativa. Sin duda alguna, se trata de un verdadero proceso cultural creativo que no puede ser estudiado solo desde el punto de vista del alumno o del docente. 
Este proceso rebasa a la simple aplicación, "a ciegas", de un programa único elaborado fuera de la práctica educativa.

Parafraseando el epígrafe de este artículo, deseamos comentar que en la nueva concepción psicológica planteada por Galperin, los fenómenos psíquicos no son substancias cristalizadas, sino que constituyen el objeto de estudio psicológico bajo la metodología formativa que permite descubrir las características de la acción humana a través del proceso de su formación desde el inicio mismo, desde el plano externo hacia el plano interno, a través de la compleja vía de transformaciones cualitativas, a la cual Galperin denominó como la concepción de la formación de las acciones por etapas. "La concepción de la formación de las acciones y conceptos mentales por etapas se ha aprobado en la práctica en su aplicación efectiva en la enseñanza escolar, así como en otras formas de educación. En el plano psicológico general, la base de esta concepción es la nueva metodología del estudio psicológico que reconstruye de manera global las representaciones tradicionales acerca de la psique, acerca del objeto y el método en la psicología” (Voiskunskiy; Zhdan; Tikhomirov, 2009, p. 356). De esta forma, consideramos que existen grandes potenciales de la concepción de la formación por etapas que aún no se han abordado.

\section{Conclusiones}

1. La teoría de Galperin y Talizina de la formación de las acciones por etapas requiere de una nueva re-conceptualización en la psicología contemporánea.

2. Esta teoría se debe comprender, no como una secuencia rígida de planos únicos, sino como una posibilidad heurística y dinámica que apoya al proceso formativo.

3. La teoría de la formación de las acciones por etapas puede utilizarse en diferentes edades en niños sanos y en niños con dificultades. 
4. La palabra orientación es la palabra calve de la teoría de Galpeirn

y Talizina e implica un cambio paradignático en la psicología general, educativa y clínica.

\section{Referencias}

ALVES, A.; GONZÁLEZ, F.; SOLOVIEVA, Yu. Avaliação do desenvolvimento a partir da psicologia histórico-cultural: enfrentando a patologização. In: GONÇALVES, M.; SANCHES, N.; PROENÇA, M. (orgs.) Avaliação psicológica e escolarização: contribuições da psicologia histórico-cultural. Teresina: EDUFPI, 2018. p. 215-246.

BONILLA, R.; SOLOVIEVA, Yu. Evidencias de la formación de la función simbólica a través de la actividad de juego de roles sociales. Cuadernos Hispanoamericanos de Psicología, Bogotá, v. 16, n. 1, p. 29-40, 2016. https://doi.org/10.18270/chps..v16i1.1967

DAVIDOV, V. V. Tipos de generalización en la enseñanza. Moscú: Sociedad Pedagógica de Rusia, 2000.

GALPERIN, P. Ya. La actividad psicológica como ciencia objetiva. Moscú: Instituto de Ciencias Pedagógicas y Sociales, 1998.

GALPERIN, P. Ya. Psicología. 4 conferencias. Moscú: Casa de Libros "Universidad", 2000.

GONZÁLES, C.; SOLOVIEVA, Yu. Impacto del juego de roles sociales en la formación de la función simbólica en preescolares. Revista de Psicología de la Universidad de Antioquía, Medellín, v. 8, n. 2, p. 49-70, 2016.

KARPOV, Yu. V.; TALIZINA, N. F. Criterios para el diagnóstico del desarrollo intelectual. Psychological Assessment, Washington, v. 2, n. 4, p. 3-18, 1986.

KARPOV, Yu. V.; TALIZINA, N. F. Psicodiagnóstico del desarrollo cognoscitivo de los escolares. Moscú: Universidad estatal de Moscú, 1989.

LEONTIEV, A. N. Obras psicológicas escogidas. Moscú: Universidad Estatal de Moscú, 1983.

LEONTIEV, A. N. Actividad, conciencia, personalidad. México: Cartago, 1984.

LEONTIEV, A. N. Génesis de la actividad. In: LEONTIEV, A. N. Formación de la psicología de la actividad. Moscú: Sentido. Serie: Clásica viva, 2003. p. 373-385.

PETUKHOV, V. V. El problema de la acción consciente. In: VOISKUNSKIY, A. E.; ZHDAN, A. N.; TIKHOMIROV, O. K. (orgs.). Tradiciones y perspectivas de la aproximación de la actividad en psicología. Moscú: Sentido, 2009. p. 235-262. 
PIAGET, J. How children learn mathematical concepts. Scientific American, New York, v. 189, n. 5, p. 74-79, 1953. https://doi.org/10.1038/scientificamerican1153-74

PODOLSKY, A. I. Prólogo. In: GALPERIN, P. Ya. Psicología. 4 conferencias. Moscú: Casa de Libros "Universidad”, 2000. p. 4-20.

PODOLSKY, A. I. La formación del concepto de número: aplicación de la metódica en una institución mexicana. In: TALIZINA, N.; SOLOVIEVA, Yu.; QUINTANAR, L. Enseñanza de las matemáticas desde la teoría de la actividad. México: CEID, 2017. p. 107-128.

RUBINSTEIN, S. L. Problemas de psicología general. St. Petersburgo: PITER, 2000. SALMINA, N. G. Signo y símbolo en la enseñanza. Moscú: Universidad Estatal de Moscú, 1988.

SOLOVIEVA, Yu. El desarrollo intelectual y su evaluación. Una aproximación histórico-cultural. México: Universidad Autónoma de Puebla, 2004.

SOLOVIEVA, Yu. La actividad intelectual en el paradigma histórico-cultural. México: CEIDE, 2014.

SOLOVIEVA, Yu. Estrategias introductorias del lenguaje escrito en el idioma inglés. México: Plaza y Valdés, 2015.

SOLOVIEVA, Yu. Enseñanza del lenguaje escrito. México: Trillas, 2016.

SOLOVIEVA, Yu.; MATA, A. Qualitative Study of Motives in Mexican School Children. Psychology Research, Wilmington, v. 7, n. 7, p. 385-396, 2017.

SOLOVIEVA, Yu.; PELAYO, H.; QUINTANAR, L. Neuropsicología de la temprana infancia. In: DA SILVA MARQUES, D. F.; ÁVILA-TOSCANO, J. H.; GÓIS, H.; LEONEL, J.; FERREIRA, N.; SOLOVIEVA, Yu.; QUINTANAR, L. (orgs.) Neuroscience to Neuropsychology: The study of the human brain. Vol. I. Barranquilla: Ed. Corporación Universitaria Reformada, 2016. p. 415-444.

SOLOVIEVA, Yu.; QUINTANAR, L. Games as a Method of Correction in Prescool Mexican Children with ADDH. British Journal of Education, Society \& Behavioural Science, London, v. 11, n. 3, p. 1-14, 2015.

https://doi.org/10.9734/bjesbs/2015/19124

SOLOVIEVA, Yu.; QUINTANAR, L. El dibujo como actividad formativa en la etapa presscolar. México: Trillas, 2016a.

SOLOVIEVA, Yu.; QUINTANAR, L. Educación neuropsicológica infantil. México: Trillas, 2016b.

SOLOVIEVA, Yu.; QUINTANAR, L. Análisis sindrómico en casos de problemas de desarrollo y aprendizaje: siguiendo a A.R. Luria. In: DA SILVA MARQUES, D. 
F.; ÁVILA-TOSCANO, J. H.; GÓIS, H.; LEONEL, J.; FERREIRA, N.;

SOLOVIEVA, Yu.; QUINTANAR, L. (orgs.) Neuroscience to Neuropsychology: The study of the human brain. Vol. I. Barranquilla: Ed. Corporación Universitaria Reformada, 2016c, p. 387-414.

SOLOVIEVA, Yu.; QUINTANAR, L. Teoría de Galperin: Orientación para psicología y neuropsicología. In: NÚÑ̃E, I. B.; RAMALHO, B. L. (orgs.). Galperin y la teoría de la formación planeada por etapas de las acciones mentales y de los conceptos. Intestigaciones y las experiencias para una enseñanza innovadora. Campinas: Mercado de Letras, 2018. p. 101-132.

SOLOVIEVA, Yu.; QUINTANAR, L. La metodología formativa en la psicología histórico cultural. Madrid: GIUNTI-EOS, 2019.

SOLOVIEVA, Yu. et al. Propuesta para el uso del juego de roles en la institución preescolar. Revista Educacao e Filosofia, Uberlândia, v. 29, n. 57, p. 153-174, 2015. https://doi.org/10.14393/revedfil.issn.0102-6801.v29n57a2015-p153a174

TALIZINA, N. F. La dirección del proceso de asimilación de conocimientos. Moscú: Universidad Estatal de Moscú, 1984.

TALIZINA, N. F. La teoría de la actividad aplicada a la enseñanza. México: Universidad Autónoma de Puebla, 2019.

TALIZINA, N. F. La teoría de la enseñanza desde la teoría de la actividad. Moscú: Universidad Estatal de Moscú, 2018.

TALIZINA, N. F.; KARPOV, Yu. V. Psicología pedagógica. Psicodiagnóstico del intelecto. Moscú: Universidad Estatal de Moscú, 1987.

TALIZINA, N. F.; KARPOV, Yu. V. Enseñanza de las matemáticas desde la teoría de la actividad. México: CEIDE, 2017.

VIGOTSKY, L. S. Obras Escogidas. Tomo 1. Madrid: Visor, 1991.

VIGOTSKY, L. S. Obras Escogidas. Tomo 3. Madrid: Visor, 1995.

VIGOTSKY, L. S. Obras Escogidas. Tomo 4. Madrid: Visor, 1996.

VOISKUNSKIY, A. E.; ZHDAN, A. N.; TIKHOMIROV, O. K. Tradiciones y perspectivas de la aproximación de la actividad en psicología. Moscú: Sentido, 2009. 